\section{Adolescência e saúde mental: revisão de artigos brasileiros publicados em periódicos nacionais}

\author{
Adolescence and mental health: a review of the \\ Brazilian literature
}

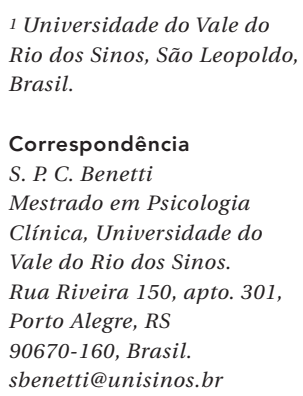

\begin{abstract}
The objective of this work was to review the Brazilian scientific literature from 1995 to 2005 on mental health in adolescence, considering the priorities identified by the World Health Organization (depression, anxiety, substance abuse, behavioral disorders, eating disorders, psychosis, child abuse, and violence). 971 abstracts were identified in the LILACS, MEDLINE, INDEXPsi, and SciELO databases, using descriptors associated with the themes. After selection according to specified criteria, 267 abstracts were analyzed by year, focus, methodological design, and principal conclusions. A qualitative analysis was performed on the main findings. Brazilian publications in this area have increased in the last five years. The majority of the articles aimed to identify the problem, while a smaller proportion dealt with the development of intervention and prevention strategies.
\end{abstract}

Adolescent; Mental Health; Review Literature
Sílvia Pereira da Cruz Benetti 1 Vera Regina Röhnelt Ramires 1 Ana Cláudia Schneider 1 Ana Paula Guzinski Rodrigues 1 Daniela Tremarin ${ }^{1}$

\section{Introdução}

O objetivo deste trabalho foi realizar um levantamento da produção nacional sobre a saúde mental na adolescência, identificando as temáticas mais freqüentes na área e destacando algumas características dos principais tópicos nesta faixa etária. O interesse em identificar essa produção justifica-se pela importância que a adolescência adquiriu nas últimas décadas, em função do reconhecimento das conseqüências negativas dos problemas de saúde mental e a constatação da menor atenção dedicada a esta faixa etária em relação às demais.

Trinta e cinco milhões de adolescentes com idade entre 10 e 19 anos foram identificados no Censo Demográfico brasileiro em 2000, realizado pelo Instituto Brasileiro de Geografia e Estatística (IBGE). Esse número, acrescido da população entre 0 e 9 anos (33 milhões), dimensiona a magnitude e a importância da necessidade de atenção à saúde mental desta faixa etária, principalmente considerando-se as estimativas de prevalência de transtornos mentais de até $20 \%$ nestes grupos 1 .

Segundo a Organização Mundial da Saúde (OMS) 2,3, algumas situações são prioridades na adolescência, tais como depressão, suicídio e psicoses. Além dessas, também devem ser considerados os transtornos de ansiedade, transtornos de conduta, abuso de substâncias, transtornos alimentares e as condições médicas associadas, 
como diabetes e epilepsias. Para uma adequada atenção a esses problemas é necessário o desenvolvimento de ações focalizando a saúde mental da criança e do adolescente baseadas na compreensão, na intervenção sobre as situações identificadas e na elaboração de diretrizes políticas.

Tomando como ponto de partida as situações prioritárias na saúde mental de adolescentes, o presente trabalho teve como objetivo principal o levantamento de artigos publicados em periódicos indexados nacionais nos últimos dez anos. Os artigos foram analisados quanto à freqüência e procedência das principais áreas de conhecimento, ao ano de publicação, ao foco de interesse (identificação, intervenções terapêuticas e programas preventivos) e às características metodológicas. Espera-se contribuir para a compreensão do problema através da identificação das características desta produção, apontando-se as necessidades identificadas como mais relevantes para o avanço do conhecimento nesta área.

\section{Considerações metodológicas}

A análise da produção científica sobre adolescência e saúde mental baseou-se na consulta às seguintes bases de dados: LILACS (Literatura Latino-Americana e do Caribe de Informação em Ciências da Saúde), Index Psi Periódicos, MEDLINE(MedicalLiteratureAnalysisandRetrieval System Online) e SciELO (Scientific Electronic Library Online). Essas bases incluem informações e referências sobre a literatura internacional e científico-técnica da América Latina e do Caribe, e fazem parte da Biblioteca Virtual em Saúde (http://www.bireme.br). Considerando-se os quadros prioritários segundo a OMS, elegeram-se as seguintes situações para investigação: depressão, ansiedade, abuso de substâncias, transtorno de conduta e delinqüência, transtornos alimentares, violência e saúde mental.

Os indexadores para a pesquisa foram selecionados segundo os Descritores em Ciência da Saúde (DeCS), agrupados em categorias de análise. Na categoria Depressão foram incluídos os resumos indexados nas palavras depressão, suicídio, transtorno depressivo; na categoria Ansiedade, ansiedade, transtorno da ansiedade, transtorno da falta de atenção com hiperatividade; na categoria Abuso de Substâncias, drogas, transtornos relacionados ao uso de substâncias; na categoria Transtorno da Conduta e Delinqüência, transtorno da conduta, delinqüência juvenil; na categoria de Transtornos Alimentares, anorexia, bulimia, transtornos alimentares; na categoria Violência, violência, violência doméstica, maustratos infantis, abuso sexual; e na categoria Saúde
Mental, psicose, transtorno psicótico, psicopatologia, saúde mental.

Inicialmente, foram levantados todos os trabalhos nacionais indexados nas bases de dados utilizando-se os limites de faixa etária (adolescência), ano de publicação (período 1995-2005) e publicação nacional brasileira. No segundo momento, excluíram-se as publicações classificadas como teses, capítulos de livros, livros, guias médicos, comentários, resenhas, informativos governamentais, mantendo-se somente os resumos de artigos que foram analisados em relação ao ano de publicação, área científica, foco de interesse, tópico principal e análise compreensiva. Artigos em língua estrangeira referentes às pesquisas brasileiras foram incluídos. Os resumos classificados em diferentes indexadores foram mantidos no principal.

\section{Resultados}

Na primeira etapa, a consulta a cada uma das bases de dados indicou um total de 129 referências na categoria Depressão, 72 em Ansiedade, 277 em Drogas/Abuso de Substâncias, 51 em Transtorno de Conduta/Delinqüência, 111 em Transtornos Alimentares, 190 em Violência/Maus-Tratos e 141 em Saúde Mental/Psicopatologia. Na Tabela 1 são apresentados os resultados iniciais em relação ao tipo de publicação e categoria. A área médica publicou $60 \%$ da produção nacional sobre saúde mental na adolescência no período 1995-2005. Seguem-se as áreas de Psicologia (22\%), de Saúde Coletiva (9\%) e de Psicanálise (4\%). Os trabalhos restantes eram provenientes das áreas de Enfermagem (3,99\%), de Odontologia $(1,89 \%)$, de Nutrição $(0,94 \%)$, de Educação Física $(0,42 \%)$, de Educação $(0,21 \%)$ e de Fonoaudiologia $(0,21 \%)$.

Na segunda etapa, a exclusão de trabalhos que não fossem artigos de periódicos específicos sobre saúde mental resultou num grupo de 267 resumos de artigos analisados quanto ao ano de publicação, área científica, foco de interesse, tópico principal, e análise compreensiva.

A produção de artigos teve um crescimento constante a partir do ano de 1995, indicando o desenvolvimento científico das questões de saúde mental (Tabela 2). Em termos de foco de interesse principal, os trabalhos abordavam a saúde mental sob a perspectiva da identificação das características individuais ou grupais, comportamentos e diagnósticos. Esse grupo constituiu $69 \%$ dos trabalhos. A área da Medicina destacouse nesse foco de interesse (45\%), visto o número de publicações sobre o diagnóstico dos transtornos mentais. 
Freqüência do tipo de publicação em relação às categorias de análise nas bases de dados Index Psi Periódicos, LILACS, SciELO.

\begin{tabular}{|c|c|c|c|c|c|c|c|}
\hline Tipo de publicação & Depressão & Ansiedade & $\begin{array}{c}\text { Transtorno de conduta/ } \\
\text { Delinqüência }\end{array}$ & Drogas & $\begin{array}{l}\text { Transtornos } \\
\text { alimentares }\end{array}$ & Violência & $\begin{array}{l}\text { Saúde } \\
\text { mental }\end{array}$ \\
\hline Artigos de revistas & 78 & 59 & 34 & 126 & 75 & 91 & 78 \\
\hline Estudos comparativos & 5 & 2 & - & 8 & 7 & 4 & 9 \\
\hline Estudos retrospectivos & 2 & - & - & - & - & - & - \\
\hline Relatos de casos & 6 & 2 & - & 3 & 6 & 9 & 9 \\
\hline Revisões & 14 & 6 & 1 & 1 & 2 & 11 & 4 \\
\hline Comentários & 1 & - & 1 & 2 & 13 & - & - \\
\hline Resenhas & 2 & - & - & - & - & - & - \\
\hline Teses & 12 & 2 & 9 & 15 & 5 & 17 & 21 \\
\hline Guias de prática médica & 4 & 1 & 1 & 4 & 2 & 8 & 2 \\
\hline Livros/Capítulos de livro & 7 & - & 3 & 95 & 1 & 42 & 16 \\
\hline Congressos & - & - & - & 8 & - & 2 & 2 \\
\hline Informativos governamentais & - & - & 2 & 15 & - & 6 & - \\
\hline Total & 131 & 72 & 51 & 277 & 111 & 190 & 141 \\
\hline
\end{tabular}

Tabela 2

Produção de artigos por ano de publicação, período 1995-2005.

\begin{tabular}{lcc}
\hline Ano & $\mathbf{n}$ & $\%$ \\
\hline 1995 & 2 & 0,7 \\
1996 & 12 & 4,5 \\
1997 & 10 & 3,7 \\
1998 & 16 & 6,0 \\
1999 & 25 & 9,4 \\
2000 & 21 & 7,8 \\
2001 & 24 & 9,0 \\
2002 & 29 & 11,0 \\
2003 & 40 & 15,0 \\
2004 & 59 & 22,0 \\
2005 & 29 & 11,0 \\
Total & 267 & 100,0 \\
\hline
\end{tabular}

Questões relacionadas aos aspectos de intervenção, incluindo estratégias psicoterápicas individuais ou grupais, serviços institucionais ou outro tipo de atendimento foram identificadas em 23\% dos trabalhos. As áreas da Medicina e Psicologia foram as que mais publicaram trabalhos sobre intervenções em saúde mental (Tabela 3). Finalmente, trabalhos sobre prevenção tiveram menor freqüência (6\%) e foram publicados predominantemente pela área de Saúde Coletiva (50\%) e respectivos aos quadros de violência e delinqüência.
Em relação à metodologia, o delineamento quantitativo descritivo foi o mais freqüente (40\%) juntamente com a categoria de artigos teóricos (40\%) acerca das situações clínicas em saúde mental. O delineamento qualitativo foi identificado em 55 investigações (22\%).

A compreensão etiológica dos transtornos mentais na adolescência apoiou-se em análises intrapessoais na maioria dos trabalhos (60\%). A inclusão de determinantes contextuais (família, escola, trabalho) ocorreu em $30 \%$ e somente 26 trabalhos (10\%) analisaram contextos macro, como política, cultura e sociedade. A compreensão intrapessoal foi mais utilizada na área da Medicina $(45 \%)$, sendo que a Psicologia (38\%) e a Saúde Coletiva (27\%) apresentaram maior número de publicações com destaque para aspectos contextuais e de interação pessoal. $\mathrm{Na}$ abordagem macro de condições política e social, a Saúde Coletiva contribuiu com $46 \%$ dos artigos e foi a área que publicou trabalhos de forma mais igualmente distribuída, evidenciando uma aproximação abrangente da questão da saúde mental.

A seguir, apresentam-se análises mais específicas das categorias avaliadas: Depressão, Ansiedade, Transtornos Alimentares, Transtorno de Conduta/Delinqüência, Transtornos associados ao Abuso de Substâncias, Psicoses e Violência, mencionando-se alguns dos trabalhos encontrados.

\section{Depressão}

Ao todo, foram identificados 40 artigos sobre o tema depressão e suicídio na adolescência. 
Distribuição por área e foco de interesse dos resumos dos artigos indexados no período 1995-2005.

\begin{tabular}{|c|c|c|c|c|c|c|}
\hline \multirow[t]{2}{*}{ Área } & \multicolumn{2}{|c|}{ Identificação } & \multicolumn{2}{|c|}{ Intervenção } & \multicolumn{2}{|c|}{ Prevenção } \\
\hline & $\mathrm{n}$ & $\%$ & $\mathrm{n}$ & $\%$ & $\mathrm{n}$ & $\%$ \\
\hline Psicologia & 36 & 19,0 & 19 & 29,2 & 4 & 25,0 \\
\hline Medicina & 84 & 45,1 & 20 & 31,0 & 4 & 25,0 \\
\hline Saúde Coletiva & 33 & 18,0 & 13 & 20,0 & 8 & 50,0 \\
\hline Psicanálise & 28 & 15,0 & 11 & 17,0 & - & - \\
\hline Outros & 5 & 3,0 & 2 & 3,0 & - & - \\
\hline Total & 186 & 100,0 & 65 & 100,0 & 16 & 100,0 \\
\hline
\end{tabular}

Quanto à distribuição da produção, verificou-se que de 1995 até 1998 a publicação aumentava em torno de 3\% em relação ano anterior. A partir daí, cresceu $10 \%$ ao ano, indicando interesse científico sobre a temática da depressão.

A maioria dos artigos publicados era proveniente da área da Medicina (62,6\%) (Pediatria e Psiquiatria, Neurologia), seguida da Psicologia com $24 \%$ dos trabalhos. A metodologia quantitativa predominou nos delineamentos $(54,1 \%)$, sendo somente três trabalhos com abordagem qualitativa e 13 artigos teóricos 4,5,6. O delineamento quantitativo caracterizou-se por estudos descritivos correlacionais 7,8,9 e estudos epidemiológicos 10,11,12,13,14.

Coerente com a metodologia descritiva, o objetivo geral era o de oferecer informações e dados clínicos acerca da depressão na adolescência. O foco prioritário era fornecer dados sobre a identificação dos indivíduos ou grupos vulneráveis. A intervenção no quadro foi abordada do ponto de vista farmacológico 15 , do exercício físico 16 , do atendimento na gestação adolescente 17 e da importância da prevenção 18 .

A depressão na adolescência foi discutida como quadro clínico em 11 artigos e nos demais em comorbidade com outros quadros, como suicídio 19,20,21, ansiedade 14, quadros neurológicos (ex. epilepsia) 22, transtorno bipolar 23 , gestação 24 , maus-tratos infantis e delinqüência, 25 e estratégias terapêuticas 26 .

Como quadro clínico, a depressão na adolescência ocorre três vezes mais freqüentemente entre os jovens do que entre os adultos 12 , cerca de 15 a 50\%, e associada a diferentes comorbidades. Entretanto, as manifestações clínicas mascaram-se por características comportamentais da fase, como agressividade, alterações do humor e sono ${ }^{4}$. Assim, o diagnóstico e a identificação específica da depressão tornaram-se uma questão básica no atendimento de saúde dos jovens 4,7, orientados por uma perspectiva multiprofissional 6,18 .

As causas da depressão incluíram abordagens contextuais que destacaram o pouco suporte familiar 7, o papel dos eventos negativos de vida 8,9, as alterações neuropsicológicas 27 e as mudanças psíquicas internas próprias da fase relacionadas às manifestações contemporâneas da cultura 28 .

\section{Ansiedade}

Dentre os 29 artigos classificados na categoria, vários focalizavam o tópico em associação a outros quadros ou situações de vida. Especificamente sobre os Transtornos da Ansiedade destacam-se artigos de revisão sobre a descrição e identificação dos sintomas, características associadas aos quadros ansiosos 29 e sobre aspectos clínicos, epidemiológicos e de intervenção 30. Outros estudos abordavam os quadros associados à ansiedade, como o transtorno da falta de atenção e hiperatividade (TDAH) discutido quanto à identificação do quadro 31 , à associação com drogas 32 , ao tratamento farmacológico 33 , à suscetibilidade genética 34 e à alta prevalência dos transtornos de ansiedade nos quadros de TDAH 35. Fundamentalmente, destacou-se a importância da identificação e do diagnóstico adequado do quadro, visto os aspectos negativos e prejuízos na aprendizagem e convívio social de adolescentes. A abordagem cognitivo-comportamental foi indicada com maior freqüência como técnica de intervenção terapêutica 36.

\section{Transtornos de conduta e delinqüência}

Um total de 27 artigos sobre transtornos de conduta e/ou delinqüência na adolescência foi identificado conforme os critérios estabelecidos. Nessa categoria, 17 trabalhos foram da área da Psicologia/Psicanálise, seis da Medicina e qua- 
tro da Saúde Coletiva. Grande parte dos trabalhos (80\%) foi composta de artigos teóricos sobre identificação de quadros clínicos 37 , sobre teorias e modelos explicativos do envolvimento com condutas agressivas 38 . Destaca-se a maior freqüência de trabalhos voltados para a discussão de modelos de intervenção e prevenção 39,40,41.

Os trabalhos de pesquisa empírica utilizaram com maior freqüência a metodologia qualitativa (33\%) e modelos compreensivos baseados em determinantes contextuais (44\%). Permaneceu, porém, o foco de interesse de identificação (56\%) de características psicológicas, familiares 37 e genéticas associadas ao comportamento anti-social 42 .

As contribuições da psicologia destacaram a precariedade das relações familiares 43 , o abandono escolar 44 , a presença de ambientes violentos 45 e também aspectos de intervenção, tais como análises do sistema socioeducativo 46 , o psicodiagnóstico interventivo 47 e trabalhos com crianças agressivas 48 . Também foram apontadas as características da cultura contemporânea 49 e da privação emocional durante o desenvolvimento 50 .

\section{Abuso de substâncias}

Ao todo 65 artigos foram classificados nesta categoria. Destaca-se que 50\% dos trabalhos foram publicados a partir de 2001. A área médica foi responsável por metade desta produção, seguida da Saúde Coletiva (27\%) e da Psicologia (19\%). Em geral, a metodologia quantitativa (53\%) predominou. Ainda assim, 23\% eram qualitativos e os restantes trabalhos teóricos fundamentados em análises documentais.

O foco de interesse centrou-se na identificação dos grupos vulneráveis e nas características de uso de drogas 51,52,53,54. As análises interpretativas basearam-se em fatores intrapessoais para o entendimento do abuso de substâncias. Entretanto, consideraram-se também aspectos interpessoais e contextuais de análise, tais como problemas escolares 55 , relações familiares 56 , sexo masculino, idade, classe socioeconômica, maus-tratos, vitimização por assaltos, presença de familiar usuário de drogas e pouca religiosidade 57,58,59. A visão psicanalítica contribuiu para a compreensão das motivações internas, o desenvolvimento psíquico e a inter-relação com representações parentais 60 .

\section{Violência}

A produção de trabalhos sobre maus-tratos e violência na adolescência aumentou a partir do ano 2000. Até aquele ano, somente 11 artigos
(33\%) dos 33 analisados sobre o tema são citados. A área da Saúde Coletiva foi a que mais publicou trabalhos sobre violência e maus-tratos (57\%), seguida da Psicologia (27,3\%) e Medicina $(15,3 \%)$. O foco de interesse das produções incluiu material sobre a identificação de casos e sobre análises teóricas baseadas em modelos de desenvolvimento (54\%). Os demais trabalhos estavam voltados para a intervenção 61 e prevenção da violência 62,63.

Além dos aspectos informativos sobre o impacto da violência e maus-tratos, os artigos focalizavam a mudança no perfil de mortalidade da adolescência e o aumento de mortes por causas externas, principalmente homicídios envolvendo jovens $64,65,66$. A violência e maus-tratos estavam associados ao comportamento sexual de risco 67 , a morar na rua 68,69 e ao desenvolvimento de condutas agressivas 70 . As repercussões negativas no desenvolvimento das situações de violência apontadas indicavam a urgência da implantação de ações preventivas e de intervenção 71,72.

\section{Transtornos alimentares}

Cinqüenta artigos discutiram os transtornos alimentares na adolescência. Igualmente às demais categorias, a publicação de trabalhos aumentou a partir de 2000 , concentrando-se $70 \%$ dos artigos entre 2000 e 2005. A maior contribuição originou-se da área medica (56\%) e Psicologia (21\%).

O foco de interesse (83\%) foi a descrição do comportamento anorético e bulímico, a identificação e prevalência dos casos 73,74,75,76, a compreensão neuropsicológica 77 e psicodinâmica do adolescente com transtornos alimentares 78 .

As intervenções nos transtornos alimentares foram abordadas por meio das experiências de equipes especializadas 79 , da clinica hospitalar 80 e de técnicas grupais 81 , psicodinâmica 82 e cognitiva 83. Predominaram discussões teóricas baseadas em compreensões das alterações da imagem corporal 84 , da dinâmica da personalidade 78 e de conflitos familiares 85 .

\section{Saúde mental}

Ao todo, 37 trabalhos foram identificados, cobrindo as áreas de Psicologia e Psicanálise (50\%), Medicina (35\%) e Saúde Coletiva (16\%). As temáticas mais freqüentes abordaram questões epidemiológicas das demandas psicológicas de instituições de atendimento ao adolescente. Nesse caso, os trabalhos tinham como foco de interesse inquéritos de morbidades em clínicas-escolas ou ambulatórios 86,87,88. Os trabalhos identificaram a alta procura de atendimento clínico por crianças e adolescentes, constituindo quase que $50 \%$ 
da clientela. A necessidade de pesquisas na área da saúde mental da infância e da adolescência e o desenvolvimento de abordagens terapêuticas focalizando serviços comunitários foram ações consideradas prioritárias para o atendimento em saúde mental 89 .

Também foram encontrados trabalhos dirigidos ao profissional da saúde 90 oferecendo informações sobre transtornos mentais na infância e adolescência. Os modelos compreensivos abordaram características interacionais de desenvolvimento e análises psicodinâmicas das mudanças da adolescência. No primeiro caso, destacavamse as etapas de desenvolvimento individual e familiar em conjunto com a exposição às situações de risco, identificando os eventos traumáticos e a resiliência individual e familiar ${ }^{91,92}$. No último caso, prevaleceram análises teóricas ou estudos de casos discutindo a compreensão psicanalítica sobre a adolescência 93,94.

Artigos sobre psicose na adolescência totalizaram oito trabalhos, metade destes organizados acerca da forma de discussão teórica sobre o adolescente psicótico 95,96,97,98, a necessidade de apoio familiar ao adolescente psicótico 99 e discussões psicanalíticas 100,101.

\section{Considerações finais}

O levantamento da produção nacional de artigos indexados em periódicos nacionais sobre saúde mental na adolescência indicou o crescimento constante da produção nacional nos últimos anos em todas as categorias investigadas, demonstrando que estão sendo reconhecidas pela comunidade científica a necessidade e a importância de investimentos de pesquisas nesta área.

Com base na análise dos resumos dos artigos também foi possível constatar diversas características desta produção. Uma observação, porém, deve ser feita quanto aos limites das generalizações do atual trabalho em relação à abrangência das indexações nas bases de dados, aos descritores utilizados, ao período selecionado para o estudo e a restrição de investigar somente estudos publicados em periódicos nacionais. Nesse último caso, verifica-se que trabalhos importantes sobre temas nacionais são publicados em periódicos internacionais. Entretanto, mesmo considerando-se esses aspectos, algumas tendências e direções podem ser observadas a partir da distribuição, foco de interesse e contribuição dos trabalhos analisados.

Em relação aos resultados, as publicações das diversas áreas indicaram que a demanda em saúde mental na adolescência é uma questão im- portante. Essa faixa etária além de constituir-se como uma grande parcela da população que procura atendimento, é identificada como um grupo etário vulnerável e de risco em todas as categorias - depressão, transtornos de conduta, transtornos alimentares, drogas e violência. Portanto, a grande demanda por atendimento e a diversidade das situações clínicas afetando os jovens são pontos fundamentais para o desenvolvimento de ações em saúde mental.

Em termos da distribuição entre as áreas de conhecimento, observa-se que grande parte das publicações provém da área médica, baseada em análises documentais ou teóricas, objetivando o fornecimento de dados epidemiológicos, informações e características clínicas para o diagnóstico das diferentes situações. Esse tipo de trabalho sinaliza o interesse pela divulgação científica dirigida aos profissionais das distintas áreas de saúde sobre os tópicos de saúde mental na adolescência, ação que se destina à qualificação, à capacitação e à divulgação do conhecimento.

De um modo geral, ainda que se observe um crescente desenvolvimento de estudos voltados para a intervenção focalizando terapêuticas e técnicas de trabalho, é pequeno o número de publicações sob este enfoque em saúde mental em todas as categorias investigadas. Cabe a ressalva que os artigos teóricos focalizando a compreensão dos quadros psicopatológicos traziam no desenvolvimento das discussões e conclusões inúmeras sugestões e indicações de fatores associados à etiologia dos transtornos mentais, apontando estratégias de intervenção e justificando a necessidade de ações preventivas. Outro ponto importante refere-se ao fato de que o interesse científico em acompanhar, avaliar e identificar o trabalho de intervenção desenvolvido na área de saúde mental da infância e adolescência está sendo consolidado. Para justificar esta análise, um exemplo seria o aumento de trabalhos sobre as clínicas-escolas de psicologia e medicina que nos últimos cinco anos têm procurado identificar as demandas clínicas no atendimento psicológico, tanto em termos individuais como de características contextuais. Igualmente, o interesse em estudos epidemiológicos da população, visando à identificação de casos e fatores associados, contribui para o desenvolvimento do conhecimento sobre as demandas em saúde mental.

Em termos metodológicos, o fato de que grande parte das publicações foi composta por resenhas teóricas e/ou estudos quantitativos descritivos, alinha-se ao objetivo básico de informar e caracterizar grupos vulneráveis ou quadros clínicos. Da mesma forma, a menor freqüência de estudos clínicos indicou que os trabalhos de 
intervenção e avaliação de programas necessitam ser ampliados.

Comparando-se a publicação nacional em relação à saúde mental independentemente de um grupo etário, Passos 102 identificou características distintas daquelas encontradas neste trabalho. Numa análise que incluiu a produção em saúde mental de 1980 a 1996, a autora identificou extensa literatura sobre análises de serviços e assistência. Esses achados não invalidam os resultados do presente trabalho e, sim, confirmam a observação de que as áreas da infância e adolescência não recebem igual atenção em relação ao adulto. E, também indicam a ênfase terciária de ação em saúde mental, que se volta quase que exclusivamente para o atendimento do indivíduo ou grupo já identificado.

\section{Conclusão}

Em síntese, pode-se concluir que a produção científica em saúde mental na faixa etária da adolescência tem crescido continuamente, característica que indica a ampliação dos estudos e investigações sobre os quadros psicopatológicos e fatores associados. Observa-se um grande interesse na compreensão e identificação dos quadros clínicos e também no delineamento de estudos voltados para o atendimento dos casos. Entretanto, mesmo considerando esses aspectos, a produção nacional em saúde mental do adolescente necessita de maior investimento nos objetivos de intervenção e prevenção. Basicamente, os atendimentos restringem-se aos casos mais necessitados e não recebem os recursos necessários para a ampliação dos serviços.

Também importante é a ampliação das investigações sobre modelos de desenvolvimento que incluam aspectos contextuais e macros de compreensão dos problemas em saúde mental, destacando as características de resiliência e saúde individual, familiar e coletiva. Essas abordagens são estratégias que permitem o desenvolvimento do conhecimento das demandas em saúde mental. Além disso, fundamentam o estudo e a adaptação dos modelos de intervenção mencionados na literatura internacional, levando em consideração as características específicas do contexto social brasileiro.

Somente a partir de sólido conhecimento, pode-se desenvolver e implantar serviços comunitários, subsidiar diretrizes, legislação e políticas públicas em saúde mental de forma que o desenvolvimento dos serviços sustente-se em apoio financeiro adequado e treinamento apropriado de recursos humanos para o trabalho.

\section{Resumo}

O objetivo deste trabalho foi realizar uma revisão da publicação nacional de artigos científicos no período de 1995-2005 sobre as situações prioritárias de saúde mental na adolescência, definidas pela Organização Mundial da Saúde, tais como depressão, ansiedade, abuso de substâncias, transtorno de conduta, transtornos alimentares, psicoses, maus-tratos e violência. Foram identificados 971 resumos nas bases de dados LILACS, MEDLINE, Index Psi e SciELO utilizando-se descritores associados ao tema. Após seleção segundo critérios, foram analisados 267 resumos científicos conforme área de produção, ano, foco, delineamento metodológico e principais resultados. A seguir, foram feitas análises qualitativas dos principais resultados. Verificou-se que a produção brasileira tem aumentado nos últimos anos e que a maioria dos trabalhos está voltada para a identificação do problema, e em menor número para o desenvolvimento de estratégias de intervenção e prevenção.

\section{Colaboradores}

S. P. C. Benetti foi responsável pela elaboração textual e análise dos resumos. V. R. R. Ramires colaborou na produção textual. A. C. Schneider, A. P. G. Rodrigues e D. Tremarin coletaram os dados bibliográficos e realizaram sínteses dos trabalhos.

\section{Agradecimentos}

Fundação de Amparo à Pesquisa do Estado do Rio Grande do Sul. 


\section{Referências}

1. Fleitlich BW, Goodman R. Implantação e implementação de serviços de saúde mental comunitários para crianças e adolescentes. Rev Bras Psiquiatr 2002; 24:2-2.

2. World Health Organization. Caring for children and adolescents with mental disorders. Setting WHO directions. Geneva: World Health Organization; 2003.

3. World Health Organization. Mental health policy and service guidance package: child and adolescent mental health policies and plans. Geneva: World Health Organization; 2005.

4. Bahls S, Bahls FRC. Depressão na adolescência: características clínicas. Interação 2002; 6:49-57.

5. Dutra EMS. Depressão e suicídio em crianças e adolescentes. Mudanças 2001; 9:27-35.

6. Versiani IM, Reis R, Figueira I. Diagnóstico do transtorno depressivo na infância e adolescência. J Bras Psiquiatr 2000; 49:367-82.

7. Baptista MN, Oliveira AA. Sintomatologia de depressão e suporte familiar em adolescentes: um estudo de correlação. Rev Bras Crescimento Desenvolv Hum 2004; 14:58-67.

8. Dell'Aglio DD, Borges JL, Santos, SS. Eventos estressores e depressão em adolescentes do sexo feminino. Psico (Porto Alegre) 2004; 35:43-50.

9. Feijó RB, Raupp APG, John AB. Eventos estressores de vida e sua relação com tentativas de suicídio em adolescentes. J Bras Psiquiatr 1999; 48:151-7.

10. Marcondes Filho W, Mezzaroba L, Turini CA, Koike A, Motomatsu Junior A, Shibayama EEM, et al. Tentativas de suicídio por substâncias químicas na adolescência e juventude. Adolesc Latinoam 2002; 3:0-0.

11. Souza E, Minayo MCS, Malaquias JV. Suicide among young people in selected Brazilian State capitals. Cad Saúde Pública 2002; 18:673-83.

12. Soares K, Almeida Filho N, Coutinho OESF, Mari JJ. Sintomas depressivos entre os adolescentes e adultos de uma amostra populacional de três centros urbanos brasileiros: análise dos dados do Estudo Multicêntrico de Morbidade Psiquiátrica. Rev Psiquiatr Clín 1999; 26:218-24.

13. Teixeira AMF, Luis MAV. Suicídio, lesões e envenenamento em adolescentes: um estudo epidemiológico. Rev Latinoam Enfermagem 1997; 5:3-6.

14. Barbosa AAG, Barbosa GA. Ansiedade e depressão na infância e adolescência: uma revisão epidemiológica e o uso de instrumentos. Neurobiologia 1999; 62:35-41.

15. Rohde LA, Tramontina S. O tratamento farmacológico do transtorno bipolar na infância e adolescência. Rev Psiquiatr Clín 2005; 32 Suppl 1:117-27.

16. Stella SG, Vilar AP, Lacroix C, Fisberg M, Santos RF, Mello MT, et al. Effects of type of physical exercise and leisure activities on the depression scores of obese Brazilian adolescent girls. Braz J Med Biol Res 2005; 38:1683-9.

17. Falcone VM, Mäder CV, Nascimento CF, Santos JM, Nóbrega FJ. Atuação multiprofissional e a saúde mental de gestantes. Rev Saúde Pública 2005; 39:612-8.
18. Sukiennik PB, Segal J, Salle E, Piltcher R, Bejzman TB, Preussler CM. Implicações da depressão e do risco de suicídio na escola durante a adolescência. Adolesc Latinoam 2000; 2:36-44.

19. Toscano JA. Depressão, suicídio e transtornos por uso de drogas na adolescência. Diagn Tratamento 2003; 8:102-4.

20. Mello MF. O suicídio e suas relações com a psicopatologia: análise qualitativa de seis casos de suicídio racional. Cad Saúde Pública 2000; 16:163-70.

21. Feijó RB, Salazar CC, Bozko MP, Bozko MP, Candiago $\mathrm{RH}$, Avila S, et al. O adolescente com tentativa de suicídio: características de uma amostra de 13 a 20 anos atendida em emergência médica. J Bras Psiquiatr 1996; 45:657-64.

22. Valente KDR, Thomé-Souza S, Kuczynski E, Negrão N. Depressão em crianças e adolescentes com epilepsia. Rev Psiquiatr Clín 2004; 31:290-9.

23. Guariglia Filho JEF, Santos IS, Scalco AZ, Castilho AR. Transtorno afetivo bipolar do tipo I na adolescência: dificuldades diagnósticas baseadas em um relato de caso. Infanto Rev Neuropsiquiatr Infanc Adolesc 2003; 10:56-62.

24. Freitas GVS, Botega NJ. Gravidez na adolescência: prevalência de depressão, ansiedade e ideação suicida. AMB Rev Assoc Med Bras 2002; 48:245-9.

25. Dell'Aglio DD, Hutz CS. Depressão e desempenho escolar em crianças e adolescentes institucionalizados. Psicol Reflex Crít 2004; 17:351-7.

26. Bahls SC, Bahls FRC. Psicoterapias da depressão na infância e na adolescência. Estud Psicol 2003; 20:25-34.

27. Laks J, Marinho VM, Rozenthal M, Engelhardt E. Neuropsicologia da depressão. Rev Bras Neurol 1999; 35:97-102.

28. Levisky DL. Depressões narcísicas na adolescência e o impacto da cultura. Psyche (São Paulo) 2002; 6:125-36

29. Castillo ARGL, Recondo R, Asbahr FR, Manfro GG. Transtornos de ansiedade. Rev Bras Psiquiatr 2000; 22 Suppl 1:20-3.

30. Asbahr FR. Transtornos ansiosos na infância e adolescência: aspectos clínicos e neurobiológicos. J Pediatr (Rio de J) 2004; 80 Suppl 1:28-34.

31. Rohde LA, Miguel Filho EC, Benetti L, Gallois C, Kieling C. Transtorno de déficit de atenção/hiperatividade na infância e na adolescência: considerações clínicas e terapêuticas. Rev Psiquiatr Clín 2004; 31:124-31.

32. Wallauer APD, Bicca CH, Salgado CAI, AlvesNeto J, Zubaran C, Pechansky F, et al. Transtorno por déficit de atenção com hiperatividade e abuso de drogas na adolescência. Rev Psiquiatr Rio Gd Sul 1996; 18 Suppl 1:162-9.

33. Segenreich D, Mattos P. Eficácia da bupropiona no tratamento do TDAH: uma revisão sistemática e análise crítica de evidências. Rev Psiquiatr Clín 2004; 31:117-23.

34. Roman T, Rohde LA, Hutz MH. Genes de suscetibilidade no transtorno de déficit de atenção e hiperatividade. Rev Bras Psiquiatr 2002; 24:196-201.

35. Souza I, Pinheiro MA, Mattos P. Anxiety disorders in an attention-deficit/hyperactivity disorder clinical sample. Arq Neuropsiquiatr 2005; 63:407-9. 
36. Ito LM, Asbahr FR. Prática da terapia cognitivocom-portamental em transtornos ansiosos na infância e adolescência. Rev Bras Psicoter 1999; 2:916.

37. Bordin IAS, Offord DR. Transtorno da conduta e comportamento anti-social. Rev Bras Psiquiatr 2000; 22 Suppl 2:SII12-5.

38. Feijó MC, Assis SG. O contexto de exclusão social e de vulnerabilidades de jovens infratores e de suas famílias. Estud Psicol 2004; 9:157-66.

39. Assis SG, Constantino P. Perspectivas de prevenção da infração juvenil masculina. Ciênc Saúde Coletiva $2005 ; 10: 81-90$.

40. Pordeus AMJ, Fraga MNO, Faco TPP. Ações de prevenção dos acidentes e violências em crianças e adolescentes, desenvolvidas pelo setor público de saúde de Fortaleza, Ceará, Brasil. Cad Saúde Pública 2003 19:1201-4.

41. Assis SG, Souza ER. Criando Caim e Abel: pensando a prevenção da infração juvenil. Ciênc Saúde Coletiva 1999; 4:131-44.

42. Kuczynski E, Gondo MCI, Rojo CRC, Assumpção JFB. Transtorno de conduta em adolescente portador de retardo mental devido à síndrome da delação do braço longo do cromossomo 18. Rev Psiquiatr Clín 1999; 26:207-9.

43. Formiga NS. A explicação dos comportamentos desviantes a partir dos indicadores da relação familiar. Psicol Argum 2004; 22:45-52.

44. Oliveira CA. A fuga da escola como preditor do fenômeno delinqüencial. Aletheia 2002; 15:27-36.

45. Oliveira MB, Assis SG. Os adolescentes infratores do Rio de Janeiro e as instituições que os "ressocializam": a perpetuação do descaso. Cad Saúde Pública 1999; 15:831-44.

46. Rauter C. Produção social do negativo: notas introdutórias. Psicol Clín 2003; 15:107-20.

47. Barbieri V, Jacquemin A, Alves ZMMB. Alcances e limites do psicodiagnóstico interventivo no tratamento de crianças anti-sociais. Paidéia 2004; 14:153-67.

48. Souza MA. Intervenção psicoterapêutica em meninos agressivos escolares como prevenção de comportamento transgressor futuro. Psicol Teor Prát 2001; 3:21-35.

49. Vilhena J, Maia MV. Agressividade e violência: reflexões acerca do comportamento anti-social e sua inscrição na cultura. Revista Mal-Estar e Subjetividade 2002; 2:27-58.

50. Sá AA. Delinqüência infanto-juvenil como uma das formas de solução da privação emocional. Psicol Teor Prát 2001; 3:13-22.

51. Tavares BF, Béria JU, Lima MS. Fatores associados ao uso de drogas entre adolescentes escolares. Rev Saúde Pública 2004; 38:787-96.

52. Baus J, Kuper E, Pires M. Prevalência e fatores de risco relacionados ao uso de drogas. Rev Saúde Pública 2002; 36:40-6.

53. Pechansky F, Szobot CM, Scivoletto S. Uso de álcool entre adolescentes: conceitos, características epidemiológicas e fatores etiopatogênicos. Rev Bras Psiquiatr 2004; 26 Suppl 1:14-7.
54. Scivoletto S, Henriques J, Gonçalves S, Andrade AG. Uso de drogas por adolescentes que buscam atendimento ambulatorial: comparação entre "crack" e outras drogas ilícitas - um estudo piloto. Rev ABP-APAL 1997; 19:7-17.

55. Tavares BF, Béria JU, Lima MS. Prevalência do uso de drogas e desempenho escolar entre adolescentes. Rev Saúde Pública 2001; 35:150-8.

56. Schenker M, Minayo MCS. A importância da família no tratamento do uso abusivo de drogas: uma revisão da literatura. Cad Saúde Pública 2004; 20:649-59.

57. Sanchez ZVM, Oliveira LG, Nappo SA. Razões para o não-uso de drogas ilícitas entre jovens em situação de risco. Rev Saúde Pública 2005; 39:599-605.

58. Sanchez ZVM, Oliveira LG, Nappo SA. Fatores protetores de adolescentes contra o uso de drogas com ênfase na religiosidade. Ciênc Saúde Coletiva 2004; 9:43-55.

59. Dalgalarrondo P, Soldera MA, Corrêa Filho HR, Silva CAM. Religião e uso de drogas por adolescentes. Rev Bras Psiquiatr 2004; 26:82-90.

60. Kessler F, Diemen L, Seganfredo AC, Brandão I, Saibro P, Scheidt B, et al. Psicodinâmica do adolescente envolvido com drogas. Rev Psiquiatr Rio Gd Sul 2003; 25 Suppl 1:33-41.

61. Ribeiro MA, Ferriani MGC, Reis JN. Violência sexual contra crianças e adolescentes: características relativas à vitimização nas relações familiares. Cad Saúde Pública 2004; 20:456-64.

62. Levisky DL. Adolescência e violência: ações comunitárias na prevenção - conhecendo, articulando, integrando e multiplicando. Ide 2002; 36:38-46.

63. Meneghel S, Armani T, Severino R, Garcia AM, Mafioleti B, Fochi E, et al. Cotidiano violento: oficinas de promoção em saúde mental em Porto Alegre. Ciênc Saúde Coletiva 2000; 5:193-203.

64. Barros MDA, Ximenes R, Lima MLC. Mortalidade por causas externas em crianças e adolescentes: tendências de 1979 a 1995. Rev Saúde Pública 2001; 35:142-9.

65. Sant'Anna A, Aerts D, Lopes MJ. Homicídios entre adolescentes no Sul do Brasil: situações de vulnerabilidade segundo seus familiares. Cad Saúde Pública 2005; 21:120-9.

66. Assis SG, Souza E. Morbidade por violência em crianças e adolescentes no Município do Rio de Janeiro. J Pediatr (Rio de J) 1995; 71:303-12.

67. Ruzany MH, Taquette SR, Oliveira RG, Meirelles $\mathrm{ZV}$, Ricardo IB. A violência nas relações afetivas dificulta a prevenção de DST/AIDS? J Pediatr (Rio de J) 2003; 79:349-54.

68. Santana JP, Doninelli TM, Frosi RV, Koller SH. Instituições de atendimento a crianças e adolescentes em situação de rua. Psicol Soc 2004; 16:59-70.

69. Menezes DMA, Brasil KCT. Dimensões psíquicas e sociais da criança e do adolescente em situação de rua. Psicol Reflex Crít 1998; 11:327-44.

70. Meneghel SN, Giugliani EJ, Falceto O. Relações entre violência doméstica e agressividade na adolescência. Cad Saúde Pública 1998; 14:327-35.

71. Brito AM, Zanetta DMT, Mendonça RV, Barison SVP, Andrade VAG. Violência doméstica contra crianças e adolescentes: estudo de um programa de intervenção. Ciênc Saúde Coletiva 2005; 10: 143-9. 
72. Gomes R, Junqueira MFPS, Silva CO, Junger WL. A abordagem dos maus-tratos contra a criança e o adolescente em uma unidade pública de saúde. Ciênc Saúde Coletiva 2002; 17:275-83.

73. Morgan CM, Vecchiatti IR, Negrão AB. Etiologia dos transtornos alimentares: aspectos biológicos, psicológicos e sócio-culturais. Rev Bras Psiquiatr 2002; 24 Suppl 1:18-23.

74. Appolinario JC, Claudino AM. Transtornos alimentares. Rev Bras Psiquiatr 2000; 22 Suppl 2:28-31.

75. Hay PJ. Epidemiologia dos transtornos alimentares: estado atual e desenvolvimentos futuros. Rev Bras Psiquiatr 2002; 24 Suppl 3:13-7.

76. Dunker KLL, Philippi ST. Hábitos e comportamentos alimentares de adolescentes com sintomas de anorexia nervosa. Rev Nutr 2003; 16:51-60.

77. Duchesne M, Mattos P, Fontenelle LF, Veiga H, Rizo L, Appolinario JC. Neuropsicologia dos transtornos alimentares: revisão sistemática da literatura. Rev Bras Psiquiatr 2004; 26:107-17.

78. Colucci RB. De quem é o desejo? A reconstrução do desejo na anoréxica. Rev Bras Psicanál 2004; 38:285-307.

79. Pinzon V, Gonzaga AP, Cobelo A, Labaddia E, Belluzzo P, Fleitlich-Bilyk B. Peculiaridades do tratamento da anorexia e da bulimia nervosa na adolescência: a experiência do PROTAD. Rev Psiquiatr Clín 2004; 31:167-9.

80. Silva MR. Anorexia nervosa em crianças e adolescentes: uma experiência clínica hospitalar. Mudanças 2003; 11:21-40.

81. Bó DEB, Barbosa R. Anorexia, bulimia e família: uma experiência com trabalho em grupo. J Bras Psiquiatr 1999; 48:533-7.

82. Gorgati SB, Holcberg AS, Oliveira MD. Abordagem psicodinâmica no tratamento dos transtornos alimentares. Rev Bras Psiquiatr 2002; 24 Suppl 1:44-8.

83. Abreu CN, Cangelli Filho R. Anorexia nervosa e bulimia nervosa: abordagem cognitivo-construtivista de psicoterapia. Rev Psiquiatr Clín 2004; 31:17783.

84. Saikali CJ, Soubhia CS, Scalfaro BM, Cordems TA. Imagem corporal nos transtornos alimentares. Rev Psiquiatr Clín 2004; 31:164-6.

85. Ribeiro KMN, Busse SR. Funcionamento familiar e anorexia nervosa. Infanto Rev Neuropsiquiatr Infanc Adolesc 2002; 10:86-9.
86. Schoen-Ferreira TH, Silva DA, Farias MA, Silvares EFM. Perfil e principais queixas dos clientes encaminhados ao Centro de Atendimento e Apoio Psicológico ao Adolescente (CAAA) - UNIFESP/EPM. Psicol Estud 2002; 7:73-82.

87. Lauridsen EP, Tanaka OY. Morbidade referida e busca de ajuda nos transtornos mentais na infância e adolescência. Rev Saúde Pública 1999; 33:586-92.

88. Romaro RA, Capitão CG. Caracterização da clientela da clínica-escola de psicologia da Universidade São Francisco. Psicol Teor Prát 2003; 5:111-21.

89. Rohde LA, Eizirik M, Ketzer CR. Pesquisa em psiquiatria da infância e da adolescência. Infanto Rev Neuropsiquiatr Infanc Adolesc 1999; 7:25-31.

90. Grillo E, Silva RJM. Manifestações precoces dos transtornos do comportamento na criança e no adolescente. J Pediatr (Rio de J) 2004; 80 Suppl 2:21-7.

91. Bastos ACS, Urpia ACM, Pinho L, Almeida Filho NM. O impacto do ambiente familiar nos primeiros anos de vida: um estudo com adolescentes de uma invasão de Salvador, Bahia. Estud Psicol 1999; 4:239-71.

92. Sapienza G, Pedromônico MRM. Risco, proteção e resiliência no desenvolvimento da criança e do adolescente. Psicol Estud 2005; 10:209-16.

93. Ungar V. O trabalho psicanalítico com adolescentes, hoje. Rev Bras Psicanál 2004; 38:735-49.

94. Guerra AMC. A psicanálise no campo da saúde mental infanto-juvenil. Psyche (São Paulo) 2005: 9:139-54.

95. Tengan SK, Maia AK. Psicoses funcionais na infância e adolescência. J Pediatr (Rio de J) 2004; 80 Suppl 2:3-10.

96. Ajzenberg RP. Casos-limite na adolescência. Rev Bras Psicanál 2004; 38:581-92.

97. Dias S. A inquietante estranheza do corpo e o diagnóstico na adolescência. Psicol USP 2000; 11:11935.

98. Moreira MS. A psicose maníaco-depressiva na infância e na adolescência. J Bras Psiquiatr 1996; 45:69-74.

99. Daure I. Relações familiares: suporte no tratamento de adolescentes. Psicol Clín 2001; 13:73-84.

100.Ribas MCC. Evocações de pais de adolescentes em crise. Estilos Clín 2002; 7:48-55.

101.Pisetta MAAM. Um encontro com a psicose na clínica. Psicol Argum 2002; 19:35-44.

102.Passos ICF. Cartografia da publicação brasileira em saúde mental: 1980-1996. Psicol Teor Pesqui 2003; 19:231-40.

Submetido em 30/Jan/2006

Versão final reapresentada em 14/Jan/2007

Aprovado em 09/Fev/2007 